\title{
A Meta-Model of Cyber-Physical-Social System: The CPSS paradigm to support Human-Machine collaboration in Industry 4.0
}

\author{
Bereket Abera Yilma ${ }^{1,2}$, Hervé Panetto ${ }^{2}$, Yannick Naudet ${ }^{1}$ \\ 1 Luxembourg Institute of Science and Technology (LIST), Luxembourg \\ 1 Universite de Lorraine, CNRS, CRAN, France \\ \{bereket.yilma, yannick.naudet\}@list.lu, herve.panetto@univ-lorraine.fr
}

\begin{abstract}
The $4^{\text {th }}$ industrial revolution (Industry 4.0) heavily relying on the concept of Cyber-Physical Systems (CPS) has transformed the manufacturing industry into an intelligent environment. Advances in manufacturing and automation industries created hyper-connected industrial ecosystems that are not limited to smart production but also facilitate organizational integration. Hence, fostering the creation of collaborative, networked and intelligent industries. One of the emerging advances in the digital transformation of industries is the creation of environments where humans work in close collaboration with sensor enabled smart machines and robots. Particularly the close involvement of humans in such smart environments challenges system designed methodologies mainly because human aspects are not considered in CPS design frameworks. In this paper, we present an approach to support this aspect of Industry 4.0 taking a CyberPhysical-Social System (CPSS) paradigm to incorporate human aspects with the existing notion of CPS. We propose a meta-model of CPSS that can serve as a framework to design systems involving human and CPS collaboration.
\end{abstract}

Keywords: Industry 4.0, Cyber-Physical-Social system, Meta-model, HumanMachine collaboration.

\section{Introduction}

The introduction of Cyber-physical systems (CPS), together with advances in Information and communication technologies (ICT) has been the major driving force for the 4th industrial revolution [10]. These advances are empowering an era of digital transformation by offering connectedness and intelligent computation. Thus, promoting collaboration in production systems and organizational integration. Industry 4.0 particularly in manufacturing and automation fields forecasts promising solutions for the future of digitized industrial ecosystem. One of the major expected outcomes of this revolution is the allocation of tedious and repetitive tasks to intelligent machines and robots [8, 12]. According to [9] "CPS describes a broad range of network connected, multi-disciplinary, physically-aware engineered systems that integrate embedded computing (cyber) and technologies into the physical world. Such design approaches mainly incorporate the orchestration of physical devices and phenomena with computational nodes". The era of digital transformation is evolving faster than expected. One aspect of this evolution is to enhance advanced collaboration 
mechanisms in industries; particularly collaborations involving humans and machines $[6,7,11,13]$. In these industrial contexts, the nature of relations between humans and CPS is demanding not only task execution but also cognitive interaction. However, the current design approaches of industrial systems heavily rely on the core concept of CPS lacking efficient means to link technical and social prospects. This degrades the quality of collaboration and can compromise safety [5] resulting challenges to deliver and cope with the speed of evolution in Industry 4.0. Particularly the main challenge originates from the complexity of human nature, as people usually do not follow rules that are not matching with their way of thinking, preferences, needs and capabilities. Additionally each individual is unique and her behaviour under different circumstances is driven by a complex phenomenon, which has not yet been fully understood.

As Industry 4.0 is enhancing such collaborative industrial environments, the need for a design framework that goes beyond CPS is not far-fetched. Hence, a system design methodology that incorporates human aspects will be complementary to the future of digitized industrial ecosystem. In this work, we propose an approach to support this prospect by taking a Cyber-Physical-Social system (CPSS) paradigm. CPSS is an emerging concept developed by integrating a social aspect to the existing CPS notion. We first conceptualise the notion of CPSS through a definition that is grounded on a generic framework provided by the theory of systems. Then we illustrate all kinds of relationships that may occur between systems, subsystems and components in a system of CPSSs. Ultimately we present a meta-model of Cyber-Physical-Social systems, which can serve as a base framework for the designing of complex systems that involve close collaboration of humans and CPS for the future Industry 4.0 applications. Finally we provide a case study example to demonstrate how the extended meta-model can be used to obtain a compact model, which can then be used for further analysis.

\section{Cyber-Physical-Social System (CPSS)}

From a general perspective CPSS is the composition of Cyber-Physical System (CPS) and Social system. CPS refers to a generation of systems with integrated computational and physical capabilities strongly related to the 4-th Industrial Revolution [1]. The Social aspect refers to interacting individuals, having each their own cognition, preferences, motivation and behaviour [2, 4]. The development of CPSS is still in its infancy. Over the past decade, different researchers used different terminologies to refer to the integration of the human aspect into CPS projecting different conceptualizations. For instance in $[16,17,18]$ Cyber-physical-human systems (CPHS) was used, being defined as "a system of interconnected systems (computers, cyber-physical devices, and people) 'talking' to each other across space and time, and allowing other systems, devices, and data streams to connect and disconnect." In [15] the concept of CyberPhysical-Social-Thinking hyperspace (CPST) was introduced for geological information service system. This work defined CPSS as "a system deployed with emphasis on humans, knowledge, society, and culture, in addition to Cyber space and Physical space. Hence, it can connect nature, cyber-space, and society with certain rules.", where as for CPST, it is established through the mergence of a new dimension 
of thinking space into the CPS space. The thinking space is a high-level thought or idea raised during the intellectual activities of people. The work visualises the Intellect of humans separately from the social aspect of CPSS as thinking space. On the other hand the term Social-Cyber-Physical-Systems (SCPS) was also used in [19] and [20], being defined as "a complex socio-technical system in which human and technical aspects (CPS) are massively intertwined." According to this definition, the scope of SCPS extends to the intangibles of social context, which includes social culture and norms, personal beliefs and attitudes, and informal institutions of social interactions. The notion of CPSS is conceived in many works; however, the usage is not homogeneous. Furthermore, the perspective and way of defining also varies from domain to domain. In an effort to address this gap, recently a holistic definition and domain -independent conceptualisation of CPSS grounded on a generic framework provided by the theory of system was proposed by [2]. In our work, we adopt a perspective on CPSS represented by the following definition.

Definition 1: CPSS is a system strictly composed of a Cyber-Physical System (CPS) and a Social System (SS), in which the system's components interact in a virtual and physical environment, where CPS and SS are defined respectively as follows.

A CPS is a system encompassing all the systems and subsystems of Cyber and Physical Systems, their components and the interaction between them, as well as integration of computation with physical processes.

A Social System is a system that comprises interacting individuals, having each their own cognition, preferences, motivation and behaviour.

Digitized industrial ecosystems emerging as a result of rapid advances in Industry4.0 are environments where humans and sensor enabled smart devices cohabit a physical space of collaboration. Hence, can be seen as CPSS environments. Following this, we present a meta-model of CPSS that can support such collaborative system design approaches in Industry 4.0.

\section{A Meta-Model of CPSS}

In this section, we illustrate all kinds of relationships that may occur between CPSSs, CPSs, SSs and their components (cyber, physical, Social). Then we ultimately formalise the CPSS paradigm through a meta-model inspired by the one proposed by [1] for CPS.

\subsection{Towards a meta-model for CPSSs}

We define a CPSS as a set CPSS $=\left\{\boldsymbol{C S}, \boldsymbol{P S}, \boldsymbol{S S}, R^{P}, R^{C}, R^{S}\right\}$, where $\boldsymbol{C S}$ (Cyber System), $\boldsymbol{P S}$ (Physical System) and $\boldsymbol{S} \boldsymbol{S}$ (Social System) are sets containing Cyber components, Physical components and Social components respectively. Atomic CPSS is one that is strictly composed of one Cyber, one Physical and one Social component. A Composite CPSS is one that is formed by the combination of CPSSs or components from two or more CPSSs. Similarly, we define CPS as a set CPS $=\left\{\boldsymbol{C S}, \boldsymbol{P S} R^{P}, R^{C}\right\}$. Atomic CPS 
is one that is strictly composed of one Cyber and one Physical component. A Composite CPS is one that is formed by the combination of CPSs or components from two or more CPSSs. Finally, $R^{P}, R^{C}, R^{S}$ represent respectively the sets of physical, cyber and social relationships that may exist between system's components or between systems parts and their environment as detailed below and illustrated in Figure 1.

$\mathbf{R}^{\mathbf{P}}$ (Physical relations):- refers to the relation between systems, subsystems and components to be physically connected and the transmission of physical objects between components.

$\mathbf{R}^{\mathbf{C}}$ (Cyber /Virtual relation):- refers to the presence of an information flow /control or sharing of computational node between/within systems, subsystems and components.

$\mathbf{R}^{\mathbf{S}}$ (Social relation):- refers to the flow of information/ transfer of knowledge between social components (i.e. humans). It also reflects cognitive ties that govern human behaviour.

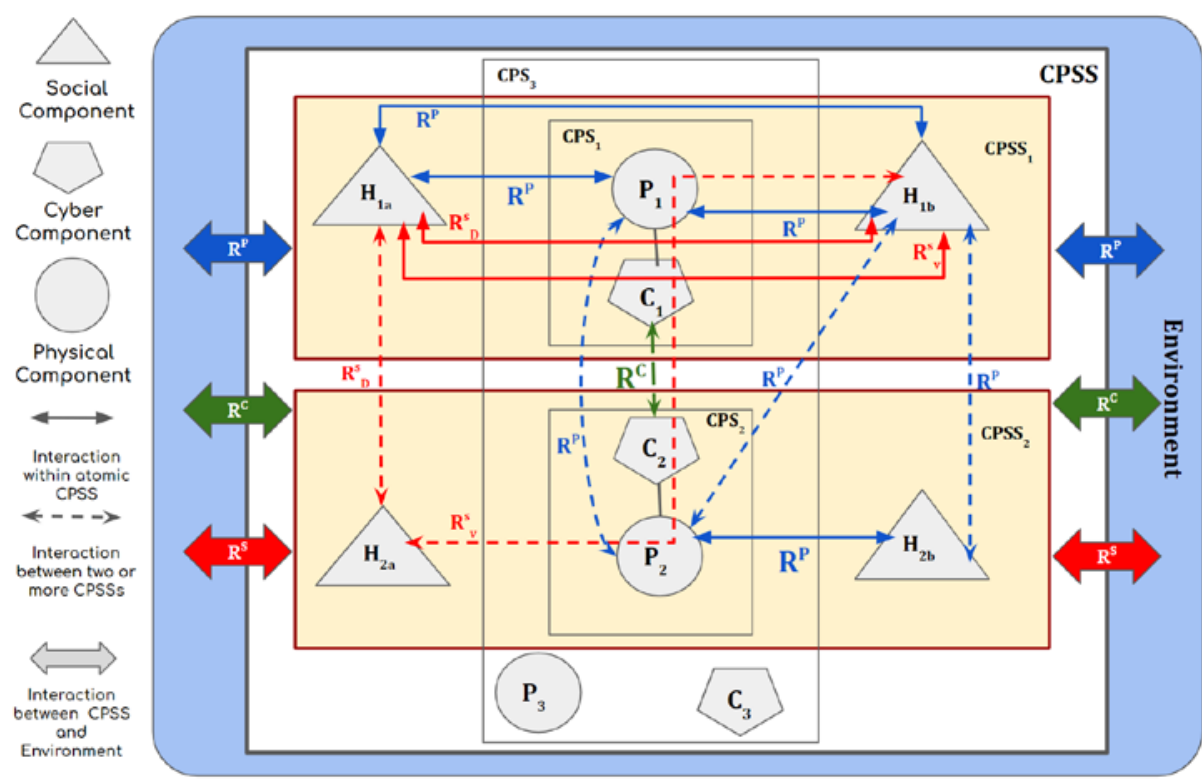

Fig. 1. A composite CPSS

Figure 1 is an example of a system of CPSS, which is composed of two CPSSs and one CPS. This example illustrates all types of relationships and forms of interactions that may exist within a system of CPSS. The top shaded box represents a single CPSS $\left(\mathrm{CPSS}_{1}\right)$, which is composed of a CPS ( $\mathrm{CPS}_{1}$, most inner box), and a Social System with two components (humans) $\mathrm{H}_{1 \mathrm{a}}$ and $\mathrm{H}_{1 \mathrm{~b}}$. $\mathrm{CPS}_{1}$ is composed of a Physical component $\left(\mathrm{P}_{1}\right)$ and a Cyber component $\left(\mathrm{C}_{1}\right)$. CPSS $\mathrm{S}_{2}$ is another CPSS containing $\left(\mathrm{CPS}_{2}\right.$, $\mathrm{H}_{2 \mathrm{a}}, \mathrm{H}_{2 \mathrm{~b}}, \mathrm{P}_{2}, \mathrm{C}_{2}$ ) for the corresponding components in $\mathrm{CPSS}_{1}$. CPS 3 is a composite CPS 
containing $\mathrm{CPS}_{1}, \mathrm{CPS}_{2}$ and its own Cyber and Physical components $\mathrm{P}_{3}, \mathrm{C}_{3}$. The shaded boarder represents the external environment.

In a system of CPSS three forms of interaction can occur. I. Interaction within a single CPSS (among subsystems and components), II. Interaction between two or more CPSSs, and III. Interaction between CPSS and its environment.

I. Interactions within a single CPSS: - interactions within a single CPSS can be of Physical, Cyber or Social nature as illustrated on figure 1.

$\mathbf{R}^{\mathbf{P}}$ : - Physical relations within a single CPSS can exist between Human and Human $\left(\mathrm{H}_{1 \mathrm{a}}\right.$ and $\left.\mathrm{H}_{1 \mathrm{~b}}\right)$, Human and Physical components $\left(\mathrm{H}_{1 \mathrm{a}}\right.$ and $\left.\mathrm{P}_{1}\right)$ and $\left(\mathrm{H}_{1 \mathrm{~b}}\right.$ and $\mathrm{P}_{1}$ ) or between two or more physical components. This type of interaction is visually observable as it involves direct contact in the physical world.

$\mathbf{R}^{\mathbf{C}}$ : - Cyber relations within a single CPSS refer to the sharing of computational resources and the flow/control of information among the different components of the system.

$\mathbf{R}^{\mathbf{S}}$ : - A social relationship within a within a single CPSS takes two different forms.

i. $\quad$ A direct communication/ Conversation in the physical world $\left(R_{D}^{S_{D}}\right)$

ii. Indirect communication through a CPS medium $\left(R_{V}^{S}\right)$.

II. Interactions between different CPSSs: - can be of Physical, Cyber or social relations represented by dotted lines on figure 1 .

$\mathbf{R}^{\mathbf{P}}$ : - A direct physical contact between Humans $\left(\mathrm{H}_{1 \mathrm{~b}}\right.$ and $\left.\mathrm{H}_{2 \mathrm{~b}}\right)$, physical components $\left(\mathrm{P}_{1}\right.$ and $\left.\mathrm{P}_{2}\right)$ or Human and Physical components $\left(\mathrm{H}_{1 \mathrm{~b}}\right.$ and $\left.\mathrm{P}_{2}\right)$.

$\mathbf{R}^{\mathbf{C}}$ : - Is a virtual information flow/ control between cyber components of two or more CPSSs $\left(\mathrm{C}_{1}\right.$ and $\left.\mathrm{C} 2\right)$.

$\mathbf{R}^{\text {S: }}$ - A social relationship between different CPSSs also takes two different forms as it is within a single CPSS (direct communication - $R_{D}^{S}$ between $\left(\mathrm{H}_{1 \mathrm{a}}\right.$ and $\left.\mathrm{H}_{2 \mathrm{a}}\right)$ and through a CPS medium - $R_{V}^{S}$ between $\left(\mathrm{H}_{1 \mathrm{~b}}\right.$ and $\left.\mathrm{H}_{2 \mathrm{a}}\right)$.

III. Interactions between CPSS and external environment: - Physical, Cyber and Social relations represented by bold arrows connecting the CPSS to different systems, subsystems and components of the external environment.

\subsection{CPSS Meta model}

In Figure 2, we propose a meta-model for CPSS based on our definition of CPSS and the formulation of a system of CPSSs, using the UML 2.0 notation. The meta-model formalizes the minimum requirement for the emergence of a CPSS as an atomic unit and the modeling of a composite CPSS. The emergence of a CPSS requires a connection between at least one social component and one physical component that is linked to at least one cyber component. 


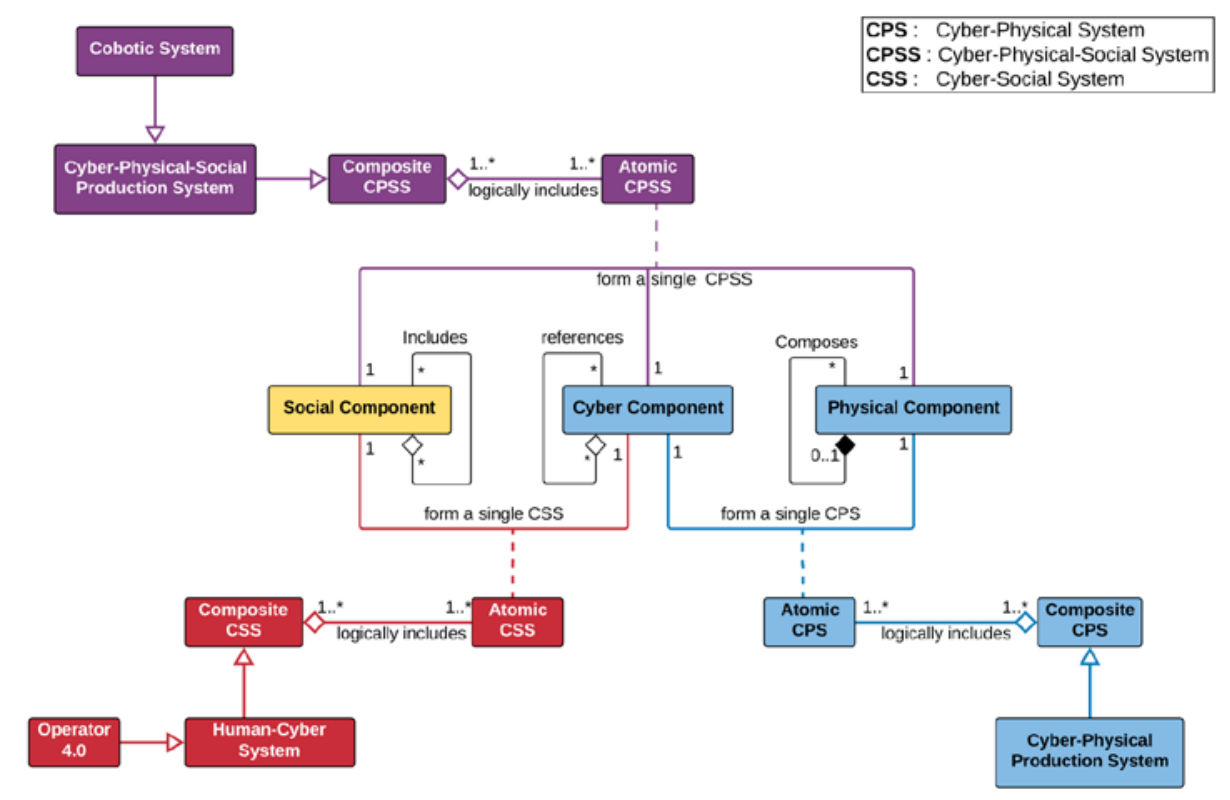

Fig. 2. Meta-model of CPSS: The blue concepts depict concepts from [1]

Atomic CPSS emerges when this minimum requirement is fulfilled. Accordingly, each atomic CPSS is given a single social component, a single physical component, which models its mechanical behaviour and a single cyber component for computational functionality. This is presented in the meta-model by the relationship 'form a single CPSS' between the classes 'Social component', 'Cyber component' and 'Physical component'. As an extension to the composition of complex CPSS the aggregate relation 'logically includes' is introduced to the model. An atomic CPSS 'is physically part of' a composite CPSS and with inheritance relation it can lend its functionality. The class of 'Cyber-Physical-Social production system (CPSPS)' can be viewed in the model as a subclass of composite CPSS and Cobotic system is a subclass of CPSPS. Whenever a system is in the relation 'is physically part of' this also entails that it is being 'logically included' in that system. A similar analogy was used in [1]. When the 'Social component' is not instantiated, an atomic CPS can emerge represented by the relationship 'form a single CPS'.

The meta-model we presented in this work is a partial extension of [1] and is subject to further extension /modification in an effort to model complex properties and interdependence between components. Additionally we introduce the notion of Cybersocial system (CSS) in the model, which is not a concept developed currently but possibly in the future. Despite the terminology, CSS is currently being used to refer an online social network, in this context the future CSS could imply a system where human cognition is supported through cyber components. This concept is also partially shared 
by [3] automation aiding enhanced cognitive capabilities such as perception, memory, reasoning and decision (Operator 4.0), a subclass of Human-Cyber system. Once having a well-developed and operational CSS, there will be a different horizon and additional possibilities to the emergence of the future CPSS. This is introduced here with the goal of having a meta-model that is flexible and open to accommodate new concepts in the evolution of the domain. However as this is not the scope of this paper, it will not be discussed further. The concept of SPS (Social-Physical system) is not included in the model because it is the long existing standard manual system of operation with no actual relevance in this context. In the following section, we present a case study of Cyber-Physical-Social production system.

\section{Case Study}

Let us consider an abstract scenario of aerospace engine systems plant modified and adopted from [14]. This case study is chosen because it simulates the emerging advent in Industry 4.0, which is a collaborative production systems between human and CPS. This is a plant producing parts of engine system for the aerospace industry. The production system is organised as job shop. In the production line of the plant, there are manufacturing engineers, operators and maintenance technicians that are skilled and able to perform tasks on different machines. In order to improve efficiency robotic cells are introduced at job shops to collaborate with the workers. In this production system there are several machines designated for different tasks. Most of the activities require similar set of skills but there are few activities that require additional skills for which additional training is needed. However, the company's orientation for task identification and worker involvement follows socio-technical principles, which allows collaboration and knowledge sharing between the workers through live chat and virtual assistance. This highlights social interaction between the workers to support each other.

Figure 3 illustrates a simplified version of this sophisticated process of the plant in an effort to visualise with best possible detail the composition of such complex CyberPhysical-Social Production System (CPSPS) and the interaction between different components. As it can be seen on the figure, one can assume a simplified version of the job shop with three different machines equipped with robotic cells to collaborate with the workers. The physical parts of each machine and robotic cells with their embedded computational capabilities form the three CPS units. However since human workers are present at the vicinity of these workstations to work in collaboration with the robotic cells, individual CPSSs can emerge at each workstation.

The current approaches of designing such systems rely on CPS frameworks, which limits the nature of collaboration to task execution leaving no room for possible cognitive interaction. In this particular case study, the introduced robotic cells are programmed to perform a predefined task and they execute it with maximum effectiveness in order to help the human workers. The collaboration in this case is only task execution or workload sharing with the humans for a global objective of improving 
performance.

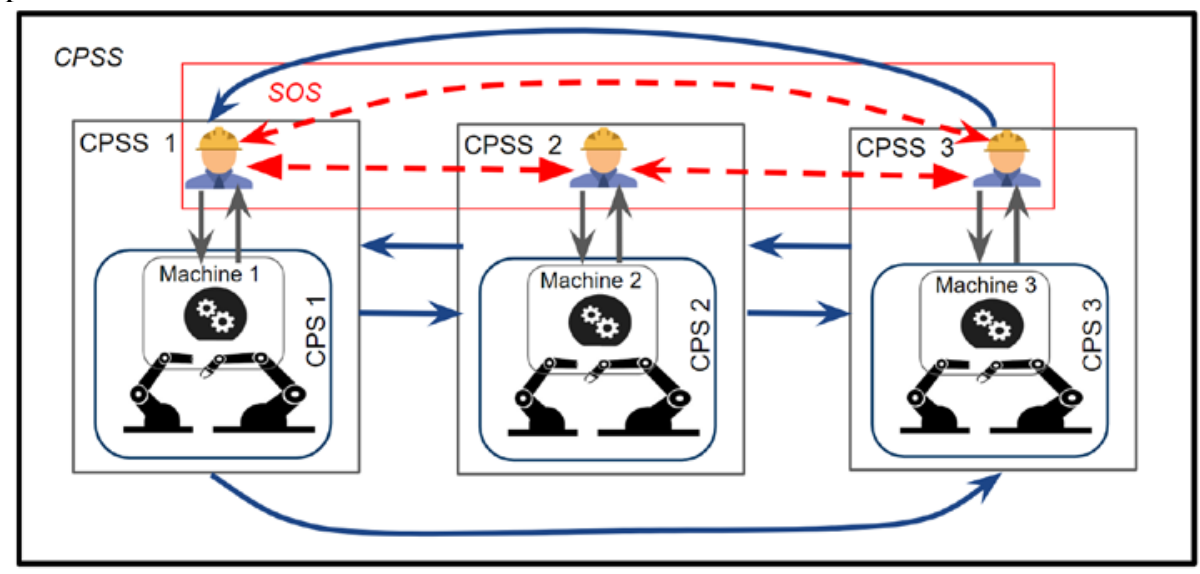

Fig. 3. Example of Cyber-Physical-Social Production System (CPSPS) applied to a plant that produces parts of engines for aerospace industry. Dotted lines represent social relations and solid lines represent Cyber and physical relations.

However, this nature of collaboration is not able to handle human dynamics (unexpected human actions and changing needs due to a different state of mind). This is unknown to the CPS, which has no capabilities to reason on such kind of changes. Hence, it can significantly affect collaboration quality, work efficiency and can compromise worker's safety. We believe collaborations in such systems should evolve from task execution to cognitive interaction. Our Postulate is that taking the CPSS paradigm opens opportunities to reconfigure such systems and put human dynamics at the center of the interaction. Such systems should be designed taking into account not only cyber and physical components but also important social aspects of humans. We believe the CPSS paradigm with the support of artificial intelligence brings several opportunities for the future of Industry 4.0 facilitating seamless collaboration and support between human and CPS.

The field of artificial intelligence is revolutionising many domains empowering systems with the ability to learn from data, reason (predict/infer), make intelligent decisions and self-correct. Industry 4.0 has unexploited potential to benefit from artificial intelligence since current manufacturing environments are equipped with sensor enabled wearables and machines capable of collecting various types of data, high speed and real time computational power. Particularly in Cyber-Physical-Social Production Systems putting human as part of the system, AI can help in transforming industry of the future to a better collaborative working environment where collaborative robots and machines can learn and recognise complex human behaviour, response patterns, values and needs. Thus, pursue important human aspects, make decisions and perform predicted actions that humans would approve to become better companions. Additionally it makes it possible to customise and personalise workspaces matching physical and cognitive characteristics of workers to support special needs, disabilities, aging and other kinds of personal limitations and opportunities. In future studies we 
aim to further develop and extend the meta-model also validate proposed opportunities at design phase through experiment at execution phase in selected case studies.

\section{Conclusion}

In this paper, we proposed an approach to support human-machine collaboration in Industry 4.0 through a Cyber-Physical-Social systems (CPSS) paradigm. CPSS is an emerging concept that intertwines human aspects to the existing notion Cyber-Physical systems (CPS). Current system designs approaches in Industry 4.0 heavily rely on the core concept of CPS, which does not incorporate human aspects. Thus, degrading collaboration quality and leaving room for compromised safety. We believe the CPSS paradigm supports the future of Industry 4.0 in bridging this gap as it effectively integrates human aspects with CPS. In general, the emergence of such intelligent collaborative industrial environments together with the complexity of human dynamics make systems more complex. Nevertheless taking a CPSS paradigm offers opportunities to better understand and model complex interactions. We elaborated the emerging notion of CPSS by visualising all kinds of relationships and interactions that may occur within a system of CPSSs. Additionally the extended meta-model is a step towards having a generic framework. As it brings human dynamics closer to CPS, it jointly opens opportunities to benefit from artificial intelligence in learning complex human behaviours, values and needs to enable machines and robots in becoming better companions during collaboration. Future studies will investigate this further.

\section{References}

1. Lezoche, M., Panetto, H.: Cyber-Physical Systems, a new formal paradigm to model redundancy and resiliency. Enterprise Information Systems. 1-22 (2018), doi: 10.1080/17517575.2018.1536807.

2. Yilma, B.A., Naudet, Y., Panetto, H.: Introduction to Personalisation in CyberPhysical-Social Systems. On the Move to Meaningful Internet Systems: OTM 2018 Workshops Lecture Notes in Computer Science. 25-35 (2019), doi: 10.1007/978-3030-11683-5_3

3. Romero, D., Bernus, P., Noran, O., Stahre, J., Fast-Berglund, Å.: The Operator 4.0: Human Cyber-Physical Systems \& Adaptive Automation Towards HumanAutomation Symbiosis Work Systems. IFIP Advances in Information and Communication Technology Advances in Production Management Systems. Initiatives for a Sustainable World. 677-686 (2016).

4. Naudet, Y., Yilma, B.A., Panetto, H.: Personalisation in Cyber Physical and Social Systems: the Case of Recommendations in Cultural Heritage Spaces. 2018 13th International Workshop on Semantic and Social Media Adaptation and Personalization (SMAP). (2018), doi: 10.1109/smap.2018.8501890.

5. Moulières-Seban, T., Bitonneau, D., Salotti, J.-M., Thibault, J.-F., Claverie, B.: Human Factors Issues for the Design of a Cobotic System. Advances in Intelligent Systems and Computing Advances in Human Factors in Robots and Unmanned Systems. 375-385 (2016). 
6. Hernoux, F., Nyiri, E., Gibaru, O.: Virtual reality for improving safety and collaborative control of industrial robots. Proceedings of the 2015 Virtual Reality International Conference on ZZZ - VRIC 15. (2015).

7. Panetto, H., Iung, B., Ivanov, D., Weichhart, G., Wang, X.: Challenges for the cyberphysical manufacturing enterprises of the future. Annual Reviews in Control. (2019).

8. Mourtzis, D., Fotia, S., Boli, N., Vlachou, E.: Modelling and quantification of industry 4.0 manufacturing complexity based on information theory: a robotics case study. International Journal of Production Research. 1-14 (2019).

9. Derler, P., Lee, E.A., Tripakis, S., Törngren, M.: Cyber-physical system design contracts. Proceedings of the ACM/IEEE 4th International Conference on CyberPhysical Systems - ICCPS 13. (2013).

10. Arnold, C., Kiel, D., Voigt, K.-I.: How The Industrial Internet Of Things Changes Business Models In Different Manufacturing Industries. International Journal of Innovation Management. 20, 1640015 (2016).

11. Bouffaron, F., Dupont, J.-M., Frédérique, M., Morel, G.: Integrative construct for Model-Based Human-System Integration: a case study. IFAC Proceedings Volumes. 47, 12317-12324 (2014)

12. Moeuf, A., Pellerin, R., Lamouri, S., Tamayo-Giraldo, S., Barbaray, R.: The industrial management of SMEs in the era of Industry 4.0. International Journal of Production Research. 56, 1118-1136 (2017).

13. Zhang, F., Liu, M., Shen, W.: Operation modes of smart factory for high-end equipment manufacturing in the Internet and Big Data era. 2017 IEEE International Conference on Systems, Man, and Cybernetics (SMC). (2017).

14. Fantini, P., Pinzone, M., Taisch, M.: Placing the operator at the centre of Industry 4.0 design: Modelling and assessing human activities within cyber-physical systems. Computers \& Industrial Engineering. (2018).

15. Y. Zhu, Y. Tan, R. Li, and X. Luo.: Cyber-physical-social thinking modeling and Computing for geological information service system. in Proceedings of the $4^{\text {th }}$ International Conference on Identification, Information, and Knowledge in the Internet of Things (IIKI 15), Beijing, China, October 2015.

16. Sowe, S.K., Simmon, E., Zettsu, K., Vaulx, F.D., Bojanova, I.: Cyber-PhysicalHuman Systems: Putting People in the Loop. IT Professional. 18, 10-13 (2016).

17. Smirnov, A., Shilov, N., Gusikhin, O.: Cyber-physical-human system for connected car-based e-tourism : Approach and case study scenario. 2017 IEEE Conference on Cognitive and Computational Aspects of Situation Management (CogSIMA). (2017).

18. Kumar, S.A., Bhargava, B., Macedo, R., Mani, G.: Securing IoT-Based CyberPhysical Human Systems against Collaborative Attacks. 2017 IEEE International Congress on Internet of Things (ICIOT). (2017).

19. Kannisto, J., Makitalo, N., Aaltonen, T., Mikkonen, T.: Programming Model Perspective on Security and Privacy of Social Cyber-physical Systems. 2016 IEEE International Conference on Mobile Services (MS). (2016).

20. Xu, Q., Su, Z., Yu, S.: Green Social CPS Based E-Healthcare Systems to Control the Spread of Infectious Diseases. 2018 IEEE International Conference on Communications (ICC). (2018). 